\title{
Why does my diabetes make me so tired?
}

\author{
Dharshini Karuppiah ${ }^{1}$, Sivatharshya Pathmanathan ${ }^{2}$ \\ ${ }^{1}$ Diabetes and Endocrinology unit, Teaching Hospital Batticaloa \\ ${ }^{2}$ Diabetes and Endocrinology unit, National Hospital of Sri Lanka \\ Correspondence email: kdharshinik@gmail.com \\ ORCHID ID: https:/ / orcid.org/0000-0003-3546-8685
}

Copyright: This is an open-access article distributed under the terms of the Creative Commons Attribution License, which permits unrestricted use, distribution, and reproduction in any medium, provided the original author and source are credited (CC BY 4.0)

Fatigue is a common symptom of diabetes and it has a huge impact on patients' self-care and quality of life. As fatigue is a non-specific and universal symptom, frequently turn out to be a neglected complaint. Easy fatigability implies the occurrence of physical and or mental exhaustion at a level of work or stress that should ordinarily not cause such exhaustion (1). The pathophysiological mechanisms for diabetes related fatigue remain largely unknown. Although fatigue in patients with diabetes is assumed to correlate with glucose homeostasis, this symptom is not limited to uncontrolled diabetes. There could be numerous biological, physical and psychological factors contribute to diabetes related fatigue. Presence of systemic low grade inflammation in diabetes also a potential contributing factor for fatigue.

As clinicians, we are all familiar with the standard way in which we approach patients with diabetes. Often our modern medical care focused on achieving number driven targets; glycaemic targets, blood pressure targets and lipid targets etc. Unfortunately this way of approach fail to improve health outcomes and also not enable the patient to feel satisfied with the process. Therefore when dealing with diabetes related fatigue, patient involvement and empowerment are the most important than physician focused approach.

A thoughtful history and examination will help sort out the possible contributing factors for the symptom of fatigue. Unhealthy lifestyle including inappropriate dietary habit, poor sleep hygiene, lack of exercise and substance abuse are clearly associated with diabetes related fatigue. While there have been no trials in patients with diabetes, there is evidence which show that regular exercise may be an effective strategy for decreasing fatigue among patients with cancer. The possible physiologic mechanisms underlying these effects could be that both cancer and type 2 diabetes are associated with higher levels of inflammatory cytokines and exercise may have anti-inflammatory properties (2). Similarly weight loss either by low caloric diet or gastric bypass surgery has been associated with improvement in fatigue among obese individuals in both general population and diabetes. Increased consumption of fiber, omega 3 fatty acids and vegetable-based proteins, with reductions in saturated fats and calories may help to reduce low-grade inflammation and related fatigue. However, extreme caloric restriction does not appear to have a beneficial effect on cytokine profiles despite weight loss (2).
Sleep disturbances is a common yet an undetected problem in patients with diabetes. Improving glycaemic control will prevent nocturia induced awakenings and thereby help to reduce fatigue. Evidence show that continuous positive airway pressure therapy for obstructive sleep apnoea among patients with diabetes has been associated with improved sleep, as well as glycaemia and fatigue (4).

Apart from assessment of glycaemic control and screening for macro and micro vascular complications, evaluation for medication related factors causing fatigue should be kept in mind. Hyperglycaemia is a known factor for diabetes related fatigue. Equally glycaemic variability and recurrent hypoglycaemia also play a central role for this symptom. Insulin and sulphonylureas are the most common culprits for hypoglycaemia especially when there is a mismatch between meal intake and treatment regimes. Statins, antihypertensives, beta-blockers and diuretics are the other potential cause for drug related fatigue in patients with diabetes.

Meticulous care should be taken to exclude other medical illnesses contributing to diabetes related fatigue such as anaemia, heart failure, renal failure and rheumatologic conditions. Patients suffering from other endocrinopathies including thyroid disorders, hypo and hypercalcaemia, vitamin D deficiency, hypogonadism and Cushings syndrome can also present with non-specific symptoms especially fatigue. There should be a high degree of clinical suspicion, but a good clinician would easily recognise the clues for these illnesses by a sensible history, examination and focused laboratory investigations.

Living with a chronic disease like diabetes is challenging for both patients and family. Inability to cope with the demands of diabetes care and the economic burden of medication cost further impair the physical and mental well being. Moreover the prevalence of depression is high among patients with diabetes, which requires proper evaluation and treatment.

To date, there are substantial evidence regarding lifestyle and behavioural therapy for diabetes related fatigue. However, only very few data available on pharmacological intervention for fatigue related to diabetes. Increased production of proinflammatory cytokines were linked to type 2 diabetes and insulin resistance, especially among obese patients, which is associated with high fatigue levels (5). These pro inflammatory cytokines are the primary therapeutic target for evolving pharmacological intervention for diabetes 
related fatigue. Disease modifying anti-rheumatic drugs have been shown to decrease fatigue and improve physical and psychological function in patients with various rheumatalogical disorders. Similarly data from few small studies show promising results with anti-inflammatory drugs among patients with diabetes ${ }^{(6)}$. Nevertheless safety and the long term benefits of such agents need further long term studies.
Health care workers involving diabetes care should understand that diabetes and fatigue are interrelated, forming a vicious cycle by worsening each other. Lifestyle interventions resulting weight loss, awareness and treatment of sleep disorders and depression, regular physical activity may reduce fatigue. Since physical and mental wellbeing and thereby quality of life is impaired by this symptom, patients presenting with the complaint of fatigue should not be neglected and need focused evaluation.

\section{References:}

1. Sanjay Kalra. Rakesh Sahay. Diabetes Fatigue Syndrome. Diabetes Ther2018; 9:1421-1429.

2. Cynthia Fritschi, Anne M. Fatigue in Adults with Type 2 Diabetes—an Overview of Current Understanding and Management Approaches. US Endocrinology 2012;8(2):84-87.

3. Julie Lasselin, Sophie Laye, Sandra Dexpert, Agnès Aubert, Concepción González, et al.. Fatigue symptoms relate to systemic inflammation in patients with type 2 diabetes. Brain, Behavior, and Immunity, 2012;26 (8): pp.1211-1219.

4. Hassaballa HA, Tulaimat A, Herdegen JJ, Mokhlesi B, The effect of continuous positive airway pressure on glucose control in diabetic patients with severe obstructive sleepapnea, Sleep Breath, 2005;9(4):176-80.

5. Lasselin J, Laye S, Dexpert S, et al., Fatigue symptoms relate to systemic inflammation in patients with type 2 diabetes, Brain Behav Immun, 2012;26(8):1211-9.

6. Cavelti-Weder C, Furrer R, Keller C, et al., Inhibition of IL-1beta improves fatigue in type 2 diabetes, Diabetes Care, 2011;34(10):e158 